\title{
JOB CHARACTERISTIC BELIEFS AND PERSONALITY AS ANTECEDENTS OF SUBJECTIVE PERSON-JOB FIT
}

\author{
Karen Holcombe Ehrhart
}

San Diego State University

\begin{abstract}
Theory and research on Person-Environment (P-E) fit tend to focus on the outcomes of fit, while antecedents have received less attention. Two antecedents of individuals' subjective Person-Job (P-J) fit are studied: personality and job characteristic beliefs (JCBs). In a framework similar to work on objective fit, the interaction of these person and environment variables is hypothesized to predict subjective P-J fit. The hypotheses are studied with customer service job scenarios that varied in the nature of employee-customer contact. Two studies found significant interactions between personality and JCBs in predicting subjective $\mathrm{P}-\mathrm{J}$ fit. Implications and directions for research are discussed.
\end{abstract}

KEY WORDS: Person-Job fit; subjective fit; job characteristics; personality.

Person-Environment (P-E) fit has been the subject of much theoretical and empirical attention over the course of several decades. Indeed, Saks and Ashforth (1997) called the topic "a cornerstone of industrial/organizational psychology and human resources management" (p. 395). Multiple conceptualizations of P-E fit exist, involving the subjective or objective correspondence between persons and jobs, groups, organizations, or vocations. The focus of the present effort is Person-Job $(\mathrm{P}-\mathrm{J})$ fit, which has been relatively neglected in recent research despite its relevance for a variety of outcomes (Edwards, 1991).

Theory and research on P-E fit have tended to focus on outcomes of fit (e.g., satisfaction, commitment) for incumbent employees. In contrast,

Address correspondence to Karen Holcombe Ehrhart, Department of Management, San Diego State University, San Diego, USA. E-mail: KEhrhart@mail.sdsu.edu

I would like to thank Benjamin Schneider and Mark Ehrhart for their helpful comments on earlier versions of this manuscript. Study 1 was conducted as part of my dissertation research at the University of Maryland; I appreciate the support of chair Benjamin Schneider and committee members Michele Gelfand, Paul Hanges, Robert Ployhart, and Susan Taylor on that research. A portion of Study 2 was presented at the 2003 conference of the Society for Industrial and Organizational Psychology in Orlando, FL. 
the conceptual antecedents of fit and the pre-hire implications of fit have been overlooked in the literature to some extent. Understanding the antecedents of fit is not only conceptually interesting, but also useful in a practical sense for organizations that are recruiting employees. As noted by Cable and Judge (1996), if quality individuals are self-selecting out of an organization's hiring process on the basis of perceptions of insufficient fit, it is important to identify the source and the accuracy of those perceptions.

In what follows, a framework is developed regarding two antecedents of subjective P-J fit (see Figure 1) and this framework is tested in two studies. Specifically, it is hypothesized that personality interacts with beliefs regarding job characteristics to yield perceptions of P-J fit. The context for the two studies involves jobs in the service sector, an important setting given the increasing share of the economy held by service organizations (Kraut \& Korman, 1999) and the extension of these organizations beyond traditional face-to-face service delivery. In the research presented here, the antecedents of $\mathrm{P}-\mathrm{J}$ fit are studied with respect to service jobs that differ in mode of customer contact (i.e., faceto-face, telephone, e-mail).

\section{THEORETICAL FOUNDATIONS OF P-E FIT RESEARCH}

Research on P-E fit is rooted in several theories, including Murray's (1938) need-press theory, the Theory of Work Adjustment (Dawis \& Lofquist, 1984), Attraction-Selection-Attrition theory (Schneider, 1987), Holland's $(1973,1996)$ theory of vocational behavior, and interactional psychology (Schneider, Smith, \& Goldstein, 2000; Terborg, 1981). These theories share an interest in the role of both person and situation in contributing to behavior, and they propose that the correspondence or fit between individuals and their environments yields positive psychological consequences.

Theory and research on $\mathrm{P}-\mathrm{E}$ fit have involved various levels of analysis with respect to the environment (e.g., jobs, organizations,

Figure 1

Research Framework

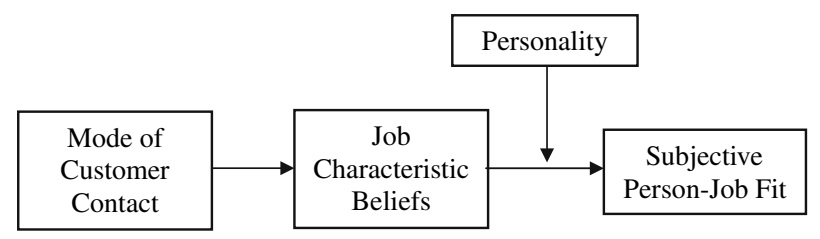


vocations). In industrial/organizational psychology and related fields, research has tended to focus on $\mathrm{P}-\mathrm{O}$ fit, with less attention to other types of fit such as P-J fit. Even studies of job choice have often involved organizational variables (e.g., pay systems, human resource systems) as the basis for fit judgments, thus invoking the concept of $\mathrm{P}-\mathrm{O}$ fit rather than P-J fit (e.g., Bretz \& Judge, 1994; Cable \& Judge, 1994). Indeed, Judge and Cable (1997) noted the predominance of Person-Organization (P-O) fit in the literature related to job choice. Empirical studies have supported the conceptual distinction between P-J fit and P-O fit (Cable \& DeRue, 2002; Kristof-Brown, Jansen, \& Colbert, 2002; Lauver \& KristofBrown, 2001), which supports the possibility that individuals can perceive a greater level of fit with their organizations than with their particular jobs, or vice versa. Since people apply for and work in specific jobs within organizations, research on both types of fit is important. Thus, the present study represents renewed attention to the construct of $\mathrm{P}-\mathrm{J}$ fit.

\section{PERSON-JOB FIT}

Fit can be evaluated subjectively or objectively (Kristof, 1996). Subjective P-J fit refers to individuals' perceptions regarding how well they fit with a particular job. For example, employees may be asked the degree to which they feel their job matches their preferences or needs. Objective P-J fit, on the other hand, pertains to how well individuals' reported preferences or characteristics correspond to a job's characteristics. For instance, job seekers' preferences may be compared with incumbent employees' evaluations of job characteristics. As noted by Judge and Cable (1997), the lack of research on subjective fit has contributed to "substantial gaps in establishing the nomological network around the construct of $\mathrm{P}-\mathrm{O}$ fit" (p. 384). Although this statement refers to $\mathrm{P}-\mathrm{O}$ fit, reflecting the recent emphasis on this type of fit, it also applies to $\mathrm{P}-\mathrm{J}$ fit. The present research represents an effort to address this stillexisting deficiency in the literature by investigating two antecedents of subjective P-J fit: job characteristic beliefs (JCBs) and personality.

The focus here on subjective perceptions of fit is based on the premise that individuals' own evaluations of fit are a more proximal predictor of pre-hire or post-hire outcomes (e.g., attraction, satisfaction) than objective fit. There is some support for this idea in the literature on job or organization attraction. A number of studies have found that person and environment characteristics interact to predict attraction, which demonstrates the influence of objective fit. However, subjective fit can be considered an unseen mediator in this research, such that the interaction between person and environment characteristics leads to perceptions of fit, which in turn predict attraction. This mediating 
relationship has been demonstrated in a couple of studies (Cable \& Judge, 1996; Dineen, Ash, \& Noe, 2002), but in general, inadequate attention has been paid to the consideration of environmental characteristics from the individual's perspective. One exception is a study by Judge and Cable (1997), who investigated P-O fit based on the congruence between individual and organizational values as reported by job applicants. The present research takes a similar approach, in that individual and job characteristics are measured from the individual's point of view, to investigate whether these predict subjective $\mathrm{P}-\mathrm{J}$ fit. The framework adopted is therefore similar to that of an objective fit study, in that person and job characteristics should interact to predict fit, but with an interest in subjective $\mathrm{P}-\mathrm{J}$ fit.

\section{JOB CHARACTERISTIC BELIEFS}

Sproull (1981) posited that "prior beliefs that organizational participants have about their environments influence their perception of actions in those environments and their responses to those actions" (p. 216). The existence and importance of such beliefs is not limited to organizational participants, however, as evidenced in a study of applicants' pre-hire organizational culture beliefs during the recruitment process by Cable, Aiman-Smith, Mulvaney, and Edwards (2000). Whereas Cable et al. focused on the implications of pre-hire beliefs regarding organizations, the focus of the current study involves perceptions of fit with regard to jobs. Accordingly, beliefs with regard to job characteristics are proposed as an antecedent of subjective P-J fit. Thus, the concept of JCBs is introduced here and defined as individuals' expectations or perceptions regarding the attributes of a particular job.

People may have JCBs related to a variety of jobs, including jobs they have experience with as well as jobs they have never held. Using reasoning similar to signaling theory, which suggests that applicants' judgments of attraction are developed based on available information about a job (e.g., Rynes, 1991; Spence, 1973), individuals' JCBs are developed based on available information about a particular job, whether or not that information is complete. Just as individuals organize and use available information about a job in determining their attraction, a similar process should occur with respect to evaluations of fit. The present research focuses on the contribution of three types of JCBs to perceived fit with three hypothetical service jobs.

\section{Job Characteristic Belief Dimensions Used in the Present Study}

The selection of the JCB dimensions for the present study was guided by a desire for (1) continuity with the job characteristics literature 
as well as (2) relevance to the context of the present research (i.e., customer service). The second goal is important in that service-oriented jobs have certain features that distinguish them from goods-oriented jobs (Bowen \& Schneider, 1988). In particular, Soteriou and Chase (1998) noted the importance of customer contact as a key differentiating feature between services and manufacturing contexts. As Stone and Gueutal (1985) observed, existing taxonomies are limited in the scope of job characteristics included and do not necessarily reflect the full set of attributes along which individuals evaluate jobs. In order to satisfy both of the above objectives, one JCB dimension was created for the present research based on the existing job characteristics literature, and two additional dimensions were developed to correspond to the context of the present study.

A fundamental taxonomy in the job characteristics literature is Hackman and Oldham's (1980) Job Characteristics Model, which includes five job characteristics (i.e., autonomy, skill variety, task feedback, task identity, task significance). Research on the model has suggested that either a multiplicative or additive index of the five job characteristics, reflecting job complexity, is a better predictor of the model's psychological outcomes than any one job characteristic by itself (Fried \& Ferris, 1987). Thus, for the sake of parsimony, a single Complexity JCB dimension was created for the present research, involving the extent to which a job is complex, challenging, and involves variety.

The five dimensions in the Job Characteristics Model largely pertain to an individual's experience of the job, independent of other parties such as subordinates, coworkers, supervisors, or customers. Accordingly, the level and nature of interpersonal interaction required by customer service jobs is not represented in the Job Characteristics Model. Indeed, the majority of the tasks included in O*NET's (2005) description of a customer service representative job pertain to interacting with others. Therefore, two additional JCB dimensions were created to represent the people-oriented and interactive nature of the jobs included here. The first, Customer Interaction, involves the frequency and quality of interactions with customers as part of the job. This dimension refers to whether the job is people-oriented and interactive with regard to dealing with customers. The second, Organizational Interaction, pertains to the frequency and quality of interactions among members of the organization. This reflects the extent to which the work environment is friendly and involves interactions with co-workers.

In summary, the three JCB dimensions used in the present research were designed to provide some continuity with the job characteristics literature as well as to capture important aspects of service jobs. Two additional considerations led to the use of this set of JCB dimensions. 
First, these dimensions could be theoretically linked to the personality dimensions described below; these conceptual connections yielded Hypotheses 2-5. Second, the three dimensions should vary across the customer service jobs considered, since a test of the interaction between personality and JCBs requires variance on both sets of variables. This expected variation is the basis for Hypothesis 1, which is developed next.

\section{JCBs in the Context of Customer Service Jobs}

Research on service jobs is important given that over the past half century, service has come to dominate the U.S. economy (Kraut \& Korman, 1999). Indeed, this trend is expected to increase. According to the Bureau of Labor Statistics (Hecker, 2004), employment in service occupations is projected to grow $20.1 \%$ from 2002 to 2012. In recent years, an increasing number of modes of communication have been adopted with respect to customer contact; it is no longer necessary for customers to be physically present in order for service encounters to take place. Advances in technology have challenged service providers to adopt new ways of doing business, and customers can often access a service employee-or the service itself-not only by going to the organization in person, but also by telephoning or e-mailing the organization or using its website. These developments in customer service work have yielded variety in the nature of customer service jobs, such that a job involving face-to-face service will be somewhat different from a job involving service provided via telephone or e-mail. Accordingly, it is expected that individuals will hold different beliefs regarding the characteristics of service jobs that involve different forms of customer contact (i.e., face-toface, telephone, e-mail).

It is important to note that although the three jobs included in the present research varied only in terms of the mode of communication used to interact with customers, this manipulation was expected to yield differences in each of the JCB dimensions measured. Two of Kellogg and Chase's (1995) dimensions of customer contact, intimacy and information richness, are helpful in explaining some of these expected differences. Intimacy reflects the idea that as jobs increase in level of customer contact, they involve increasing levels of emotional intensity and require increasing levels of communication and interpersonal skills. Information richness also relates to the increasing intensity of interpersonal interaction involved in jobs with increasing customer contact. More specifically, it pertains to the nature and amount of material transmitted between the customer and service provider, which may be affected by the communication medium used. Since the three jobs in the present research involve different types of contact with customers, their corresponding differences in intimacy and in the richness of information or 
communication involved with customers should yield different levels of Customer Interaction and Complexity beliefs. In addition, the technological differences among the three jobs have implications for the level of interdependence or interaction with other members of the organization. Thus, differences in the intimacy and information richness involved in communication within the organization should yield differences in Organizational Interaction beliefs.

In short, beliefs regarding each of these three dimensions can be examined with the hypothesis that they would differ across the three jobs. No hypotheses were made regarding the form of these differences (e.g., whether Customer Interaction would be highest for jobs involving face-to-face contact). Rather, Hypothesis 1 is framed in general terms:

H1: Individuals hold different JCBs with regard to the Customer Interaction, Organizational Interaction, and Complexity of service jobs that differ in mode of customer-employee contact (i.e., face-toface, telephone, e-mail).

\section{PERSONALITY AND INTERACTIONS WITH JCBS}

As noted earlier, research on $\mathrm{P}-\mathrm{J}$ fit is strongly rooted in theories that propose an interaction between person and environment characteristics. According to these theories, an individual's fit is based on the correspondence or match between the attributes of individual and the environment. With regard to subjective $\mathrm{P}-\mathrm{J}$ fit, the focus of the current research, this means that individuals take into account their own characteristics as well as an evaluation of the job's characteristics when developing perceptions of fit with the job. The set of individual characteristics selected for the present studies is personality in terms of the Five-Factor Model (FFM). This taxonomy has been shown to generalize across cultures and across rating formats (Mount \& Barrick, 1995). Its dimensions are briefly described next to form a foundation for the hypotheses that follow (Goldberg, 1990; McCrae \& Costa, 1987; Mount \& Barrick, 1995).

The dimension of Extraversion involves attributes such as sociability, affiliation, and gregariousness as well as the extent to which individuals are dominant, bold, and assertive. Agreeableness involves a tendency to be altruistic, cooperative, and warm. Individuals high on this dimension are oriented toward serving and helping others. Emotional Stability refers to individuals who are poised, self-reliant, and stable, although it is often discussed in terms of Neuroticism, referring to individuals low on Emotional Stability. Neuroticism involves character- 
istics such as insecure, indecisive, and tense. Openness to Experience has sometimes been considered to represent intellect, and has also been discussed as involving culture. Attributes included in this dimension include imaginative, curious, and original. Finally, a primary component of the Conscientiousness dimension is dependability. Individuals high on Conscientiousness tend to be responsible, organized, and efficient.

Although a sizeable amount of research has used the FFM to predict variables such as job performance (e.g., Barrick \& Mount, 1991) and job satisfaction (e.g., Judge, Heller, \& Mount, 2002), and some of this research has incorporated job characteristics (e.g., Mount, Barrick, \& Stewart, 1998), the FFM has seldom been studied with regard to P-E fit. One exception is a study by Judge and Cable (1997) involving the relationship between personality and preference for organizational culture. These authors hypothesized and found relationships between the FFM personality dimensions and organizational culture preference. For example, individuals high on Agreeableness tended to prefer more supportive cultures and less aggressive cultures. In addition, the fit between participants' culture preferences and organizational culture as rated by other job seekers was significantly related to participants' attraction following job interviews.

In another study using the FFM, Lievens, Decaesteker, Coetsier, and Geirnaert (2001) investigated the interaction of personality and organization characteristics in predicting attraction. Results indicated that some personality dimensions interacted with organization characteristics to influence attraction. More specifically, high Openness to Experience was associated with a higher likelihood of attraction to multinational organizations, and high Conscientiousness was associated with a higher likelihood of attraction to large organizations. Although the authors framed this study as related to the literature on $\mathrm{P}-\mathrm{O}$ fit, fit was not explicitly measured in this study. Nevertheless, it is likely that subjective $\mathrm{P}-\mathrm{O}$ fit mediated the relationship between personality and organization characteristics and the outcome of attraction. That is, it is possible that the interaction between personality and organization characteristics was related to perceptions of fit, which in turn was related to attraction.

These two studies, along with similar research that did not employ the FFM (Bretz \& Judge, 1994; Cable \& Judge, 1994; Judge \& Bretz, 1992; Turban \& Keon, 1993) provide some preliminary support for Schneider, Goldstein, and Smith's (1995) assertion that "people's preferences for particular organizations are based upon an implicit estimate of the congruence of their own personal characteristics and the attributes of potential work organizations" (p. 749). The present research focuses on perceptions of fit regarding jobs, rather than organizations, with the general hypothesis being that personality interacts with JCBs to predict subjective P-J fit. 
Given that the context of the present studies involves customer service jobs, those personality dimensions that are most relevant to customer service are the primary focus of the hypotheses that follow. The literature on service-oriented personality, or service orientation, provides some insight as to the attitudes and behaviors important for customer service workers, such as courtesy, consideration, perceptiveness, and the ability to communicate accurately and pleasantly (Hogan, Hogan, \& Busch, 1984). Frei and McDaniel (1998) noted that several themes underlie service orientation measures: friendliness, responsiveness, and courteousness. Accordingly, Hurley (1998) provided theoretical and empirical evidence that Extraversion and Agreeableness are relevant to customer service jobs; Frei and McDaniel also found these dimensions to be significantly correlated with customer service measures. Extraversion and Agreeableness are therefore of primary interest in the hypotheses developed for the present research, although all five dimensions are measured in the two studies.

\section{Extraversion}

As noted earlier, those who are high on Extraversion are peopleoriented and outgoing. Judge and Cable (1997) reported that such individuals were more likely prefer a team-oriented organizational culture, presumably because of their preference for affiliation. Similarly, research on Holland's (1973) vocational interest typology has shown a positive relationship between Extraversion and Holland's Social dimension, which involves attributes such as friendly, sociable, and cooperative (Blake \& Sackett, 1999; De Fruyt \& Mervielde, 1999; Tokar \& Swanson, 1995). Thus, for individuals high on Extraversion, P-J fit should be greater with respect to jobs that are perceived to involve greater quality and quantity of interpersonal interaction with individuals inside or outside the organization. That is, as a job is perceived more people-oriented, more interactive, and involving more frequent and higher quality interpersonal interaction, people high on Extraversion should perceive better fit with the job. For those people low on Extraversion, there should either be no relationship or a negative relationship between $\mathrm{P}-\mathrm{J}$ fit and beliefs regarding interpersonal interaction. The following hypotheses are thus proposed:

H2: Extraversion interacts with Customer Interaction beliefs in predicting subjective $\mathrm{P}-\mathrm{J}$ fit such that as these beliefs increase, individuals higher on Extraversion should perceive better fit as compared to individuals lower on Extraversion. 
H3: Extraversion interacts with Organizational Interaction beliefs in predicting subjective P-J fit such that as these beliefs increase, individuals higher on Extraversion should perceive better fit as compared to individuals lower on Extraversion.

\section{Agreeableness}

Agreeableness involves characteristics such as warm and altruistic, and it refers to an orientation toward helping others. Judge and Cable (1997) found this dimension of the FFM to be positively related to preference for a supportive organizational culture and negatively related to preference for an aggressive organizational culture. Research on Holland's (1973) model has indicated a positive relationship between Agreeableness and Holland's Social dimension, which includes elements of cooperation and sociability as well as helpfulness and kindness. For individuals high on Agreeableness, then, P-J fit should be greater with respect to jobs that are perceived to involve more opportunities for interactions that involve helping or serving customers. For individuals low on Agreeableness, there should either be no relationship or a negative relationship between $\mathrm{P}-\mathrm{J}$ fit and beliefs regarding interacting with customers. Accordingly, the following hypothesis is proposed:

H4: Agreeableness interacts with Customer Interaction beliefs in predicting subjective $\mathrm{P}-\mathrm{J}$ fit such that as these beliefs increase, individuals higher on Agreeableness should perceive better fit as compared to individuals lower on Agreeableness.

\section{Other Dimensions of the FFM}

Thus far, the two personality dimensions relevant to customer service contexts have been theoretically linked to the three JCB dimensions that were designed to reflect these contexts. The remaining JCB dimension, Complexity, is expected to interact with Emotional Stability in predicting P-J fit. People high in Emotional Stability are likely to be poised and stable (as opposed to tense or insecure). Such individuals have been found to prefer innovative and decisive organizational cultures (Judge \& Cable, 1997). Findings regarding the relationship between Holland's (1973) dimensions and the FFM are inconsistent, but Hofstee, de Raad, and Goldberg (1992) found Emotional Stability to be positively related to Holland's Enterprising dimension, which can be described as energetic and self-confident. Emotional Stability may therefore interact with JCBs pertaining to the complexity, challenge, or difficulty of the job, such that individuals high on Emotional Stability would be expected to 
display positive relationships between perceived $\mathrm{P}-\mathrm{J}$ fit and beliefs that a job is challenging and complex. For individuals low on Emotional Stability, there should either be no relationship or a negative relationship between these beliefs and P-J fit. This leads to the following hypothesis:

H5: Emotional Stability interacts with Complexity beliefs in predicting subjective P-J fit such that as these beliefs increase, individuals higher on Emotional Stability should perceive better fit as compared to individuals lower on Emotional Stability.

Note that no hypotheses were created for Conscientiousness and Openness to Experience, since there were no straightforward theoretical linkages to the JCB dimensions used here.

\section{STUDY 1}

\section{Overview of Research Design}

Study 1 involved a mixed experimental design incorporating both within-subjects and between-subjects components. The within-subjects variables were participants' JCBs and subjective P-J fit with respect to three part-time customer service job scenarios which varied according to the mode of contact between customers and employees (i.e., face-to-face, telephone, and e-mail contact). The use of a within-subjects design is consistent with past research related to fit (e.g., Bretz \& Judge, 1994; Cable \& Judge, 1994; Judge \& Bretz, 1992; Kristof-Brown et al., 2002), and it represents the reality that individuals usually consider and choose from more than one job alternative. That is, individuals tend to have multiple options in the job search process, and they likely make evaluations of fit with respect to each job. The between-subjects component of the study involved individual differences in personality.

\section{Participants and Procedure}

Participants were 242 undergraduate students at a Mid-Atlantic university. The part-time customer service job scenarios used in this study were designed to be realistic for these participants. The work experience of the current sample supports the use of these scenarios. More specifically, $90 \%$ of the total sample $(N=225)$ reported some experience in a customer service job, ranging from 2 months to 20 years, with a mean of 4.3 years $(s d=4.4 \text { years })^{1}$ The sample was split fairly evenly between men and women. Age ranged from 17 to 40 years; $81.1 \%$ of students were $17-$ 23 years old. Participants were diverse in terms of ethnicity (58.7\% were European/European-American), mirroring the university population. 
In the first phase of data collection, students completed a personality measure (the NEO-FFI, described below). For most of the sample (66.5\%), this took place 8-12 weeks before the second phase of the study. The remainder of the sample $(33.5 \%)$ completed the NEO-FFI during the same session as the materials in the second phase of the study. Analyses revealed no significant differences between these groups of students, in that the pattern of results found with each group of participants was the same.

In the second phase of data collection, participants read a scenario in which they were told to consider three part-time customer service jobs at a large organization. An introductory paragraph indicated that the three jobs were for the same organization at the same location, and they had the same salary. All participants then received the same three job descriptions (the order of presentation was randomized; no effects for order were found). Each job description included a brief statement indicating the mode of customer contact (i.e., face-to-face, telephone, or email) as well as the following statement regarding job requirements: "This job involves providing a variety of services to customers, including responding to questions and handling transactions. It is important that employees treat customers with respect and courtesy at all times." The scenarios were intentionally designed to be brief, for two reasons: (1) to realistically represent the limited information that individuals possess about jobs they do not hold, and (2) to allow for maximal variability in JCBs by providing few details about the jobs. After reading each job description, participants reported their JCBs and perceptions of $\mathrm{P}-\mathrm{J}$ fit with respect to that job, then they proceeded to the next job description. The materials concluded with demographic and work experience items.

\section{Materials}

\section{Job Characteristic Beliefs}

Three dimensions of JCBs were included in Study 1: Customer Interaction, Organizational Interaction, and Complexity. The development of these items was guided by providing a pilot sample of 76 undergraduate Psychology students with job descriptions similar to those described above, and asking the students to list three adjectives that described each job. As noted earlier, these dimensions were selected to provide continuity with the job characteristics literature and relevance to the context of the present research, as well as to be theoretically connected to the personality dimensions used here.

To measure beliefs along these three JCB dimensions, a set of 14 items was created based on the pilot sample data. Participants indicated the degree to which each item characterized each service job scenario using a five-point scale ( $1=$ to little or no extent to $5=$ to a great extent). 
Based on reliability and confirmatory factor analyses, this set of 14 items was reduced to 12 items, as follows: Customer Interaction beliefs were measured with three items involving frequency of interactions with customers, interactive work, and people-oriented work. An additional item was originally included on this scale but was dropped due to reliability analyses. Specifically, alpha reliabilities ranged from .56 to .79 for the three service jobs before dropping this item, and ranged from .67 to .80 after dropping the item. Organizational Interaction beliefs were measured using two items regarding frequency and quality of interactions with organization members. An additional item was initially included on this dimension but was dropped after reliability analyses. Alpha reliabilities ranged from .58 to .69 across the three jobs when this item was included on the scale, but ranged from .71 to .76 after the item was dropped from the scale. Finally, Complexity beliefs were measured with seven items related to challenge, variety, and complexity; reliabilities ranged from .73 to .84 for the three jobs. A confirmatory factor analyses on these three dimensions yielded CFIs ranging from .95 to .97 across the three jobs and RMSEAs ranging from .05 to .06 for the three jobs. The full set of items is presented in the Appendix.

\section{Personality}

Personality was assessed using the NEO-Five Factor Inventory (NEO-FFI; Costa \& McCrae, 1992), a 60-item instrument that measures Extraversion, Agreeableness, Emotional Stability, Openness to Experience, and Conscientiousness. Participants used a five-point scale ( $1=$ strongly disagree to $5=$ strongly agree) to respond to each item . Scale scores were created by summing responses to items on each dimension; missing data were replaced with a neutral response as suggested by Costa and McCrae (1992).

The alpha coefficient reliabilities for the five scales were acceptable: .79 for Extraversion, .79 for Agreeableness, .85 for Emotional Stability, .70 for Openness to Experience, and .82 for Conscientiousness. These values are similar to or higher than the reliabilities reported in the manual for the measure (Costa \& McCrae, 1992).

\section{Subjective $P-J$ fit}

Following each job description, six items assessed subjective $\mathrm{P}-\mathrm{J}$ fit. Using a five-point scale, participants indicated the extent to which they agreed with each item ( $1=$ strongly disagree to $5=$ strongly agree). Four of these items were based on Saks and Ashforth (1997) (e.g., "The job is a good match for you," "The job would enable you to do the kind of work you want to do"). The other two items were "Your personality matches the 'personality' of the job" and "You would fit the job." The alpha coefficient reliabilities for the three job descriptions ranged from .86 to .94 . 


\section{Demographic and Work Experience Items}

Participants reported their age, gender, and ethnicity. They also indicated the number of years and/or months of experience in any job requiring customer service primarily in person, via telephone, and via e-mail (three items).

\section{Analytical Procedure}

Hierarchical linear modeling (HLM) was used to conduct the majority of the analyses. HLM is appropriate for studies that include both within-subjects and between-subjects data, and it can accommodate interactions between variables at these different levels (Hox \& Kreft, 1994). As noted earlier, in the present study the within-subjects component involved participants' responses to three service job descriptions (i.e., JCBs, subjective P-J fit), and the between-subjects component involved participants' personality data. One of the advantages to HLM is that it can take into account repeated measurements within subjects. This is important since "misestimated standard errors occur with multilevel data when we fail to take into account the dependence among individual responses" (Bryk \& Raudenbush, 1992, p. 84). In the case of the present research, dependence among responses exists since participants reported JCBs and subjective P-J fit for three different jobs.

Analyses proceeded in a two-stage process. In the first stage, withinsubjects-Level 1-relationships were calculated. These analyses indicated whether within-subjects predictors (i.e., JCBs) were significantly related to within-subjects outcomes (i.e., subjective P-J fit). The Level 1 model with respect to the effect of Customer Interaction beliefs on fit, for example, can be summarized with the following equation: $\mathrm{P}-\mathrm{J}$ fit $t_{i j}=\mathrm{B}_{0 j}+$ $\mathrm{B}_{1 j}\left(\right.$ Customer Interaction $\left.{ }_{i j}\right)+r_{i j}$, in which “ $i$ " refers to a particular job description and " $j$ " refers to an individual. The $r_{i j}$ term represents withinperson residual variance. In summary, this phase of the HLM analyses was focused only on the Level 1 variables and involved an exploratory investigation of the direct effects of JCBs on P-J fit.

In the second stage, between-subjects-Level 2-relationships were evaluated. More specifically, these analyses involved relationships between personality and the within-subjects variables (i.e., JCBs, subjective P-J fit). Two types of models were run in this phase of the HLM analyses. First, interactions between personality (between-subjects) and JCBs (within-subjects) were evaluated using "slopes as outcomes" models. These analyses tested the hypotheses involving the interaction between personality and JCBs in predicting P-J fit. For example, the interaction between Extraversion and Customer Interaction in predicting $\mathbf{P}-\mathrm{J}$ fit is represented by the following equation: $\mathbf{B}_{1 j}=\gamma_{10}+\gamma_{1 j}$ $\left(\right.$ Extraversion $\left._{1 \mathrm{j}}\right)+u_{1 j}$, in which $\gamma_{10}$ represents the mean of the slopes 
across individuals, $\gamma_{1 j}$ represents the slope for a particular individual, and $u_{1 j}$ represents residual variance. Non-hypothesized interactions were tested as well (e.g., Extraversion and Complexity, Openness to Experience and Customer Interaction). Second, "intercepts as outcomes" models were run to test how personality related to JCBs and P-J fit. For example, the relationship between Extraversion and Customer Interaction can be represented by the equation $\mathrm{B}_{0 j}=\gamma_{00}+\gamma_{01}$ (Extraver$\left.\operatorname{sion}_{1 j}\right)+u_{0 j}$, where $\gamma_{00}$ is the mean of the intercepts across individuals, $\gamma_{01}$ is the slope for Extraversion, and $u_{0 j}$ is the residual intercept variance. No hypotheses were made with respect to these relationships.

\section{Results}

In what follows, results pertaining to the hypothesized relationships are presented first, followed by additional results. Table 1 presents descriptive statistics and correlations.

\section{Hypothesized Results}

Hypothesis 1, that the three different service job scenarios would elicit different JCBs, was supported. As seen in Table 2, repeated measures analyses of variance indicated significant differences in mean levels of each of the JCB dimensions across the three service jobs. Followup contrast analyses revealed that for each type of JCB, the means for each of the three service jobs were significantly different from each other (see Table 2). More specifically, the face-to-face job was seen as having the highest level of each JCB dimension, followed by the telephone job, followed by the e-mail job. To some extent these analyses served as

Table 1

Means, Standard Deviations, and Correlations for Study 1, across Jobs

\begin{tabular}{lrrrrrrrrrr}
\hline & $M$ & $S D$ & 2 & 3 & 4 & 5 & 6 & 7 & 8 & 9 \\
\hline 1. P-J fit & 2.44 & 1.00 & $\mathbf{. 4 3}$ & $\mathbf{. 6 0}$ & $\mathbf{. 6 2}$ & .08 & .09 & -.01 & -.00 & .01 \\
2. Customer Int. & 3.87 & 1.18 & & $\mathbf{. 4 9}$ & $\mathbf{. 5 5}$ & -.00 & -.02 & .02 & -.04 & -.02 \\
3. Organizational Int. & 2.76 & 1.09 & & & $\mathbf{. 5 7}$ & .02 & .05 & -.02 & -.00 & -.02 \\
4. Complexity & 2.82 & .75 & & & & .07 & .09 & .01 & .00 & .02 \\
5. Extraversion & 43.32 & 6.19 & & & & & $\mathbf{. 3 2}$ &.- .46 & .02 & $\mathbf{. 2 0}$ \\
6. Agreeableness & 42.58 & 6.25 & & & & & & -.32 & .12 & $\mathbf{. 1 7}$ \\
7. Emotional Stability & 31.91 & 7.60 & & & & & & & -.06 &.- .27 \\
8. Openness to Exper. & 41.36 & 5.70 & & & & & & & & .06 \\
9. Conscientiousness & 38.20 & 5.91 & & & & & & & & \\
\hline
\end{tabular}

Note: $N=726$. Correlations in bold type are significant at $p<.05$ (based on cutoff for $\mathrm{df}=242$, due to three responses per participant on variables $1-4)$. P-J fit = Subjective P-J fit, Customer Int. = Customer Interaction, Organizational Int. = Organizational Interaction, Openness to Exper. = Openness to Experience. 
Table 2

Repeated-Measures ANOVAs and Means for JCBs, Study 1

\begin{tabular}{lcccccc}
\hline $\begin{array}{l}\text { Job Characteristic } \\
\text { Belief }\end{array}$ & $\begin{array}{c}\text { Wilk's } \\
\text { Lambda }\end{array}$ & $F(2,240)$ & Power & $\begin{array}{c}\text { Mean }(S D) \\
\text { Face-to-Face }\end{array}$ & $\begin{array}{c}\text { Mean }(S D) \\
\text { Telephone }\end{array}$ & $\begin{array}{c}\text { Mean }(S D) \\
\text { E-mail }\end{array}$ \\
\hline $\begin{array}{l}\text { Customer Interaction } \\
\text { Organizational }\end{array}$ & .24 & 381.51 & 1.0 & $4.79(.36)$ & $4.06(.84)$ & $2.77(1.10)$ \\
$\quad \begin{array}{l}\text { Interaction } \\
\text { Complexity }\end{array}$ & .42 & 166.27 & 1.0 & $3.60(.88)$ & $2.43(.89)$ & $2.25(.96)$ \\
& .39 & 189.85 & 1.0 & $3.38(.65)$ & $2.67(.64)$ & $2.42(.60)$ \\
\hline
\end{tabular}

Note: All $F$ values are significant at $p<.01$. Means within each job characteristic belief are significantly different from one another at $p<.05$ ( $\mathrm{F}$ values range from 6.34 to 766.18 , $\mathrm{df}=1,241)$.

manipulation checks, as they indicated that participants perceived the three jobs to be different from one another along all three job characteristic belief dimensions. Thus, it was logical to proceed with tests for statistical interactions.

Results with regard to the hypothesized interactions are presented in Table 3. Hypothesis 2 was supported by a significant interaction between Extraversion and Customer Interaction beliefs in predicting perceived P-J fit across the three service jobs. A plot of the interaction indicated that for individuals high on Extraversion, the relationship between Customer Interaction and $\mathrm{P}-\mathrm{J}$ fit was positive; for individuals low on Extraversion, this relationship was positive, but weaker (interaction plots are available upon request). Hypothesis 3 was supported by a significant interaction between Extraversion and Organizational Interaction beliefs in predicting $\mathrm{P}-\mathrm{J}$ fit. The nature of the interaction was similar to the one described for Extraversion and Customer Interaction. Hypothesis 4 was not supported, in that Agreeableness did not significantly interact with Customer Interaction beliefs in predicting $\mathrm{P}-\mathrm{J}$ fit. Finally, with regard to Hypothesis 5, there was a significant interaction between Emotional Stability and Complexity beliefs to predict P-J fit, but it was not in the expected direction. For individuals high on Emotional Stability, the relationship between Complexity and P-J fit was positive, as expected, but for individuals low on Emotional Stability, this relationship was also positive and slightly stronger.

Hypothesized interactions were also tested after controlling for a number of variables: age, gender, ethnicity, and work experience. Each control variable was examined by entering it into the HLM analysis at the same time as the variables representing the hypothesized relationship (e.g., age was entered with Extraversion, Customer Interaction, and the interaction between these). The pattern of results remained the same after each of the aforementioned control variables was entered. 
Table 3

Hierarchical Linear Modeling Analyses: Hypothesized Interactions, NonHypothesized Significant Interactions, and Main Effects Predicting Perceived P-J Fit, Study 1

\begin{tabular}{lcrrrr}
\hline Interaction & Parameter Value & SE & $t$ & df & $p$ \\
\hline Hypothesized Interactions & & & & & \\
Extraversion $\times$ Customer Interaction & .02 & .00 & 3.95 & 482 & $<.01$ \\
Extraversion $\times$ Organizational Interaction & .01 & .00 & 2.58 & 482 & .01 \\
Agreeableness $\times$ Customer Interaction & .00 & .00 & -.55 & 482 & .58 \\
Emotional Stability $\times$ Complexity & -.01 & .01 & -1.98 & 482 & .05 \\
Non-Hypothesized Significant Interactions & & & & & \\
Extraversion $\times$ Complexity & .02 & .01 & 3.72 & 482 & $<.01$ \\
JCBs Main Effects & & & & & \\
Customer Interaction & .39 & .03 & 14.05 & 483 & $<.01$ \\
Organizational Interaction & .58 & .03 & 21.03 & 483 & $<.01$ \\
Complexity & .89 & .04 & 22.22 & 483 & $<.01$ \\
Personality Main Effects & & & & & \\
Extraversion & .01 & .01 & 2.06 & 240 & .04 \\
Agreeableness & .01 & .01 & 1.47 & 240 & .14 \\
Emotional Stability & -.00 & .01 & -.51 & 240 & .61 \\
Openness to Experience & .00 & .01 & .05 & 240 & .96 \\
Conscientiousness & .00 & .01 & .29 & 240 & .78 \\
\hline
\end{tabular}

\section{Additional Interactions}

Besides the hypothesized interactions that significantly predicted P-J fit, post-hoc analyses revealed one non-hypothesized interaction between personality and JCBs (see Table 3). Specifically, Extraversion significantly interacted with the Complexity scale in predicting P-J fit. A plot of this interaction showed that for individuals high on Extraversion, the relationship between Complexity and $\mathrm{P}-\mathrm{J}$ fit was positive; for individuals low on Extraversion, this relationship was positive, but weaker.

\section{Main Effects}

Although the interactions between JCBs and personality were of primary interest in this research, the main effects of these two antecedents of P-J fit were also examined across the three jobs (see Table 3). Results showed that Customer Interaction, Organizational Interaction, and Complexity were positively related to fit across the three jobs. Extraversion was also positively related to $\mathrm{P}-\mathrm{J}$ fit across the three jobs.

\section{STUDY 2}

Purpose

The second study was designed to replicate and extend the findings of Study 1. Specifically, Study 2 was intended to address whether 
industry or type of organization played a role in the results found in the first study. Study 1 participants were only told that the service organization was "large." Since a variety of types of service organizations exist, it is possible that individuals' JCBs and/or P-J fit perceptions could vary as a function of the type of organization involved, and that this would affect the interactions between JCBs and personality in predicting $\mathrm{P}-\mathrm{J}$ fit. There is some empirical evidence for the usefulness of incorporating organization type in Study 2. Using a verbal protocol analysis methodology, Cable and Graham (2000) found that an organization's industry or primary business was the factor most frequently mentioned by individuals as they evaluated an organization's reputation. Moreover, Cable and Graham found industry type to be significantly related to organizational reputation perceptions in a policy capturing study of hypothetical organizations as well as in a field study of actual organizations. This suggests that more broadly, individuals may hold different impressions of work environments (in the present research, jobs) based on the type of organization involved.

To investigate whether organization type would play a role in the relationships studied in Study 1, three sets of hypothetical scenarios were used in Study 2. These scenarios differed with regard to the nature of the organization. One set of scenarios indicated that the three jobs (i.e., face-to-face, telephone, e-mail) involved a retail organization, a second set of scenarios specified a banking organization, and the third set of scenarios employed the descriptions from Study 1, in which no information about the type of organization was given. In the first two sets of scenarios, the manipulation of organization type was accomplished by inserting the word "retail" or "banking" when referring to the organization or job portrayed. As in the initial creation of the job scenarios, this manipulation was designed to be brief in order to realistically represent the limited information individuals have about jobs they do not hold.

The selection of retail and banking as contexts for Study 2 was based on a pilot study in which 84 participants were asked the following questions after reading the three job scenarios used in Study 1: "When reading one or more of the job descriptions, were you imagining a particular type of organization or job (yes or no)? If yes, what type of organization or job?" Retail and banking were the two most commonly mentioned contexts by participants who indicated that they were envisioning a particular job or organization type. The prevalence of these responses was not entirely surprising as they are contexts that participants should be familiar with as customers and/or employees. Indeed, the Department of Labor (2005) indicated retail salesperson as the most widely held occupation in 2002, with over 4 million individuals employed in such positions, and the banking industry employed approximately 1.8 million individuals in 2002. An additional advantage of using the retail 
and banking contexts is that they represent two rather distinct customer service settings.

\section{Method}

Overview of Research Design

The design of Study 2 was the same as Study 1: a mixed design incorporating both within-subjects (i.e., JCBs, subjective P-J fit) and between-subjects (i.e., personality) components. The only difference was that Study 2 involved an additional between-subjects component, as participants were randomly assigned to one of three conditions involving the context in which the job descriptions were framed.

\section{Participants and Procedure}

Participants were 216 undergraduate students at a West Coast university. $94 \%$ of the sample $(N=204)$ reported some experience in a customer service job; this experience ranged from 1 month to 25 years with a mean of 3.1 years $(s d=3.2$ years $){ }^{1}$ Approximately $2 / 3$ of the sample was female and 1/3 was male. Age ranged from 18 to 43 years; $94.9 \%$ of students were 18-23 years old. Participants were quite diverse in regard to ethnicity (57.0\% were European/European-American), consistent with the university population.

Measures in Study 2 were administered to participants in a single session. As in Study 1, participants were presented with a hypothetical scenario in which they were told to consider three part-time customer service jobs at a large organization. As noted above, Study 2 also included the manipulation of organization type: retail $(N=71)$, banking $(N=72)$, or none specified $(N=73)$.

\section{Materials}

The measures of personality, subjective P-J fit, demographics, and work experience were the same as those used in Study 1. The alpha reliabilities for the personality measure were similar to those in Study 1: .79 for Extraversion, .68 for Agreeableness, .84 for Emotional Stability, .71 for Openness to Experience, and .78 for Conscientiousness. Means and standard deviations for the five personality dimensions (see Table 4) were similar to those found in Study 1. Alpha reliabilities for the P-J fit measure ranged from .92 to .97 across the three jobs.

The job characteristic belief measure was slightly modified in an attempt to improve the measurement of each dimension. Participants indicated the degree to which 17 items characterized each service job scenario using a five-point scale ( $1=$ to little or no extent to $5=$ to a great extent). One item was subsequently dropped due to reliability analyses, resulting in a set of 16 items along the three dimensions. Customer 
Table 4

Means, Standard Deviations, and Correlations for Study 2, across Jobs

\begin{tabular}{lrrrrrrrrrr}
\hline & $M$ & $S D$ & 2 & 3 & 4 & 5 & 6 & 7 & 8 & 9 \\
\hline 1. P-J fit & 2.61 & 1.09 & $\mathbf{. 4 9}$ & $\mathbf{. 5 8}$ & $\mathbf{. 4 1}$ & .07 & .04 & .03 & -.01 & .00 \\
2. Customer Int. & 3.42 & 1.17 & & $\mathbf{. 7 3}$ & $\mathbf{. 4 5}$ & .05 & .02 & -.01 & .02 & .05 \\
3. Organizational Int. & 3.05 & 1.00 & & & $\mathbf{. 4 5}$ & .08 & -.02 & -.03 & -.01 & .05 \\
4. Complexity & 3.09 & .68 & & & & .04 & .07 & .07 & -.05 & -.01 \\
5. Extraversion & 42.52 & 6.04 & & & & & .23 & -.32 & .01 &. $\mathbf{2 5}$ \\
6. Agreeableness & 43.00 & 5.31 & & & & & & -.28 & .10 &. $\mathbf{2 8}$ \\
7. Emotional Stability & 34.13 & 7.47 & & & & & & & -.05 & -.38 \\
8. Openness to Exper. & 39.85 & 5.92 & & & & & & & & -.01 \\
9. Conscientiousness & 38.76 & 5.51 & & & & & & & & \\
\hline
\end{tabular}

Note: $N=648$. Correlations in bold type are significant at $p<.05$ (based on cutoff for $\mathrm{df}=216$, due to three responses per participant on variables $1-4$ ). P-J fit = Subjective P-J fit, Customer Int. = Customer Interaction, Organizational Int. = Organizational Interaction, Openness to Exper. = Openness to Experience.

Interaction beliefs were measured with six items (e.g., frequent interactions with customers, interactive, people-oriented); alpha reliabilities ranged from .74 to .84 for the three service jobs. Four items measured Organizational Interaction beliefs (e.g., frequent interactions with organization members, quality interactions with organization members). An additional item was dropped from this scale based on reliability analyses; alpha reliabilities ranged from .63 to .80 for the three service jobs before dropping this item and ranged from .70 to .79 after dropping the item. Complexity beliefs were measured with six items (e.g., challenge, complexity, difficult); alpha reliabilities ranged from .71 to .87 for the three service jobs. A confirmatory factor analyses on these three dimensions yielded CFIs ranging from .89 to .92 across the three jobs and RMSEAs were .07 for the three jobs. The full set of items is presented in the Appendix.

\section{Analyses}

The same analysis strategy employed in Study 1 was used in Study 2: hierarchical linear modeling was used for the majority of the analyses, with the two-stage strategy described earlier.

\section{Results}

Analyses of the Study 2 data revealed similar results to those of Study 1 . In addition, analyses revealed that organization type (i.e., retail, banking, none specified) did not appear to play a role in the pattern of 
results: the relationships reported below were consistent across these three contexts. As the three organizational context conditions yielded similar patterns of results, this manipulation is not discussed further in this section. Table 4 displays the means, standard deviations, and correlations among the variables in Study 2.

\section{Hypothesized Results}

As in Study 1, Hypothesis 1 was supported (see Table 5). Repeatedmeasures analyses of variance showed significant differences in mean levels of each type of job characteristic belief across the three service jobs. Contrast analyses indicated that for each JCB dimension, the means for each of the three jobs were significantly different from each other (see Table 5). As in Study 1, the face-to-face job was rated highest on each JCB dimension, followed by the telephone job, followed by the e-mail job. These perceived differences among the jobs rendered it possible to proceed with tests for statistical interactions.

Study 2 results with respect to the hypothesized interactions are presented in Table 6. Hypotheses 2 and 3 were again supported, in that Extraversion significantly interacted with Customer Interaction and Organizational Interaction in predicting subjective P-J fit. For both of these interactions, the positive relationship between JCBs and $\mathrm{P}-\mathrm{J}$ fit was stronger for individuals high on Extraversion. For individuals low on Extraversion, this relationship was also positive, but weaker. Consistent with Hypothesis 4, Agreeableness significantly interacted with Customer Interaction beliefs to predict $\mathrm{P}-\mathrm{J}$ fit. For individuals high on Agreeableness, the relationship between this JCB dimension and $\mathrm{P}-\mathrm{J}$ fit was positive; for individuals low on Agreeableness this relationship was also positive, but not as strong. Hypothesis 5 did not receive support, however, as Emotional Stability and Complexity beliefs did not interact to predict $\mathrm{P}-\mathrm{J}$ fit.

Table 5

Repeated-Measures ANOVAs and Means for JCBs, Study 2

\begin{tabular}{lcccccc}
\hline $\begin{array}{l}\text { Job Characteristic } \\
\text { Belief }\end{array}$ & $\begin{array}{c}\text { Wilk's } \\
\text { Lambda }\end{array}$ & $F(2,214)$ & Power & $\begin{array}{c}\text { Mean }(S D) \\
\text { Face-to-Face }\end{array}$ & $\begin{array}{c}\text { Mean }(S D) \\
\text { Telephone }\end{array}$ & $\begin{array}{c}\text { Mean }(S D) \\
\text { E-mail }\end{array}$ \\
\hline $\begin{array}{l}\text { Customer Interaction } \\
\text { Organizational }\end{array}$ & .21 & 397.59 & 1.0 & $4.49(.50)$ & $3.44(.81)$ & $2.33(.93)$ \\
$\quad \begin{array}{l}\text { Interaction } \\
\text { Complexity }\end{array}$ & .28 & 278.34 & 1.0 & $3.96(.59)$ & $2.75(.79)$ & $2.43(.86)$ \\
& .61 & 67.54 & 1.0 & $3.43(.58)$ & $3.00(.63)$ & $2.84(.71)$ \\
\hline
\end{tabular}

Note: All $F$ values are significant at $p<.01$. Means within each job characteristic belief are significantly different from one another at $p<.05$ ( $F$ values range from 5.25 to 738.20 , $\mathrm{df}=1,215)$. 
Table 6

Hierarchical Linear Modeling Analyses: Hypothesized Interactions, NonHypothesized Interactions, and Main Effects Predicting Perceived P-J Fit, Study 2

\begin{tabular}{lcrrrr}
\hline Interaction & Parameter Value & SE & $t$ & df & $p$ \\
\hline Hypothesized Interactions & & & & & \\
Extraversion $\times$ Customer Interaction & .03 & .01 & 4.96 & 430 & $<.01$ \\
Extraversion $\times$ Organizational Interaction & .02 & .01 & 3.43 & 430 & $<.01$ \\
Agreeableness $\times$ Customer Interaction & .02 & .01 & 2.41 & 430 & .02 \\
Emotional Stability $\times$ Complexity & -.01 & .01 & -1.56 & 430 & .15 \\
Non-Hypothesized Significant Interactions & & & & & \\
Extraversion $\times$ Complexity & .03 & .01 & 2.91 & 430 & $<.01$ \\
Agreeableness $\times$ Complexity & .02 & .01 & 2.07 & 430 & .04 \\
Openness to Experience $\times$ Customer Interaction & .01 & .01 & 2.07 & 430 & .04 \\
JCBs Main Effects & & & & & \\
Customer Interaction & .46 & .03 & 14.82 & 431 & $<.01$ \\
Organizational Interaction & .65 & .03 & 19.12 & 431 & $<.01$ \\
Complexity & .64 & .06 & 11.19 & 431 & $<.01$ \\
Personality Main Effects & & & & & \\
Extraversion & .01 & .00 & 1.85 & 214 & .06 \\
Agreeableness & .01 & .01 & 1.06 & 214 & .29 \\
Emotional Stability & .00 & .01 & .58 & 214 & .56 \\
Openness to Experience & -.00 & .01 & -.42 & 214 & .67 \\
Conscientiousness & -.00 & .01 & -.28 & 214 & .78 \\
\hline
\end{tabular}

Each of these hypothesized relationships was tested after controlling for age, gender, ethnicity, and work experience. As in Study 1, each control variable was examined by entering it into the HLM analysis at the same time as the variables representing the hypothesized relationship (e.g., age was entered with Extraversion, Customer Interaction, and the interaction between these). The pattern of results remained the same after each of the aforementioned control variables was entered.

\section{Additional Interactions}

Three non-hypothesized interactions between personality and JCBs were found to be significant in the prediction of $\mathrm{P}-\mathrm{J}$ fit perceptions (see Table 6).

Extraversion significantly interacted with Complexity beliefs to predict subjective P-J fit. Specifically, individuals high on Extraversion showed a positive relationship between Complexity beliefs and P-J fit. For individuals low on Extraversion, this relationship was weaker, but still positive. In addition, Agreeableness significantly interacted with Complexity beliefs to predict subjective P-J fit. For individuals high on Agreeableness, these beliefs were positively related to $\mathrm{P}-\mathrm{J}$ fit; for individuals low on Agreeableness these beliefs were also positively related to 
P-J fit, but the relationship was not as strong. Finally, Openness to Experience interacted with Customer Interaction beliefs in the prediction of subjective P-J fit. For individuals high on Openness to Experience, Customer Interaction beliefs were positively related to P-J fit; for individuals low on Openness to Experience these beliefs were also positively related to $\mathrm{P}-\mathrm{J}$ fit, but the relationship was weaker.

\section{Main Effects}

As in Study 1, each of the job characteristic belief dimensions significantly predicted subjective P-J fit (see Table 6). More specifically, beliefs regarding Customer Interaction, Organizational Interaction, and Complexity were positively related to P-J fit. There were no significant relationships between personality and subjective P-J fit (see Table 6).

\section{GENERAL DISCUSSION}

The purpose of this research was to examine two possible antecedents to subjective P-J fit. The framework employed here was similar to research on objective fit, in that person and environment characteristics were proposed to interact to predict fit. However, the antecedent variables-personality and JCBs-were measured from the individual's perspective with an interest in predicting subjective P-J fit.

In two studies, customer service job scenarios that differed in the type of customer contact involved were presented to participants, who indicated their JCBs as well as their fit with respect to each job. In Study 2 , the organizational context in which the jobs were framed was manipulated, but this did not appear to have an effect on the pattern of results. In summary, JCBs along all dimensions (i.e., Customer Interaction, Organizational Interaction, Complexity) differed across the three service job scenarios presented, supporting Hypothesis 1. As hypothesized, Extraversion significantly interacted with Customer Interaction and Organizational Interaction beliefs, Agreeableness significantly interacted with Customer Interaction beliefs, and Emotional Stability significantly interacted with Complexity beliefs to predict P-J fit. Additional significant, but non-hypothesized, interactions provide further support for the idea that personality and job characteristics interact in predicting $\mathrm{P}-\mathrm{J}$ fit. The following paragraphs discuss these results and their implications in more detail.

\section{JCBs: Differences Across Jobs}

In both Study 1 and Study 2, the three customer service jobs were perceived to differ according to each of the dimensions of job character- 
istics. Not only were there significant differences among the jobs for each dimension of JCBs, but also the pattern of means was the same across both studies. It is not surprising that Customer Interaction beliefs differed across the three jobs, as the jobs were explicitly designed to differ in mode of customer contact (i.e., face-to-face, telephone, e-mail). Customer Interaction beliefs were the highest for the face-to-face job, which suggests that this job was viewed as involving the richest exchanges between customers and employees, in terms of the quality and quantity of interpersonal interaction.

It is important, however, to observe that JCBs along the other dimensions were significantly different despite the limited differences among the set of jobs used in the present study. More specifically, Organizational Interaction beliefs were highest with respect to the faceto-face job; this job was likely seen as involving more opportunities for interactions with co-workers as compared to the other two jobs. Complexity beliefs also significantly differed across the jobs; the face-toface job was associated with the highest level of these beliefs, although the mean level of Complexity for the face-to-face job was just over 3 on a 5 -point scale. Perhaps the direct interpersonal interactions involved in this job are seen as more challenging than communicating over the telephone or via e-mail.

In summary, differences in mode of customer contact were found to correspond to differences along all three JCB dimensions. Not only was it important to verify that JCBs differed among the three jobs to provide a basis for testing the interaction between JCBs and personality in predicting $\mathrm{P}-\mathrm{J}$ fit, but it is also interesting to note that participants perceived differences along all of these job characteristic dimensions despite the narrow range of stimulus jobs. This suggests the usefulness of the JCB construct; even in the presence of limited information, individuals appear to hold a somewhat detailed impression of a job's characteristics.

\section{Interactions Between Personality and JCBs}

Tables 3 and 6 present results pertaining to interactions between personality and JCBs in predicting subjective P-J fit. The strongest support was found for Hypotheses 2 and 3, based on significant interactions in both studies between Extraversion and Customer Interaction as well as Organizational Interaction in predicting P-J fit. It is understandable that individuals who are more outgoing and more people-oriented would perceive higher levels of fit with jobs that they believe provide better and more frequent interpersonal interactions with customers or organization members. For individuals low on Extraversion the relationships between these beliefs and $\mathrm{P}-\mathrm{J}$ fit were not as strong, although they were still positive. It appears, therefore, that interper- 
sonal interaction is a valued characteristic of jobs, especially for individuals high on Extraversion.

In addition, support was found in Study 2 for Hypothesis 4, involving the interaction between Agreeableness and Customer Interaction beliefs in predicting perceptions of $\mathrm{P}-\mathrm{J}$ fit. It makes sense that individuals who have a more cooperative and altruistic orientation would perceive a greater degree of fit with jobs that they perceive to involve interacting with and serving customers. For individuals lower on Agreeableness the relationship between these beliefs and $\mathrm{P}-\mathrm{J}$ fit was positive as well, although not as strong. This finding provides support for the notion that individuals are likely to report fit with jobs that allow them to help and serve others, particularly if their level of Agreeableness is high. Given the support for this hypothesis in Study 2, it is somewhat surprising that similar results were not found in Study 1. It is possible that the items that were added to the Customer Interaction scale in Study 2 played a role in this difference in results. For instance, the addition of the item involving satisfying interactions with customers and the reverse-scored item regarding the job being impersonal may have struck a chord with individuals higher in Agreeableness and contributed to the stronger relationship between this JCB dimension and fit for these individuals. In contrast, the set of items in Study 1 may not have reflected the quality of customer interactions as much as the quantity of these interactions. That is, perhaps what is appealing to those higher in Agreeableness is the nature of the interactions with customers and not just the amount of such interactions.

A significant interaction between Emotional Stability and Complexity beliefs was found in Study 1, although the nature of this interaction was different from what was predicted in Hypothesis 5 . Specifically, results revealed that individuals who were lower on Emotional Stability (i.e., who were less secure or stable) were slightly more likely to report that they fit with jobs with a greater level of complexity. For individuals higher on Emotional Stability, the relationship between Complexity beliefs and P-J fit was also positive, though slightly weaker. As noted earlier, the three jobs were not rated very highly on this JCB dimension, such that these jobs may have appeared as somewhat simple and routine. It is therefore understandable that people would welcome some level of challenge with respect to the jobs presented here, although the nature of the interaction is somewhat puzzling. Perhaps individuals lower in Emotional Stability were open to perceiving fit when they perceived more challenge, as long as they believed that the jobs were not excessively complex. In other words, people lower in Emotional Stability may be comfortable with complexity in a job up to a certain point, as long as the job is not viewed as too intimidating. Individuals higher in Emotional Stability may seek more complexity than is offered by this set of 
jobs, thus contributing to the weaker relationship between this JCB dimension and fit perceptions. The fact that this finding was not replicated in Study 2, however, suggests that it merits future investigation.

In addition to the significant hypothesized interactions, Studies 1 and 2 revealed other interactions between personality and JCBs as related to $\mathrm{P}-\mathrm{J}$ fit, which merit future investigation. As whole, these results suggest that dimensions of personality and JCBs interact in predicting subjective P-J fit.

\section{Independent Effects of Personality and of JCBs on Fit}

In addition to the interactions between personality and JCBs, direct relationships were tested between these variables and subjective P-J fit. The finding that Extraversion was significantly related to fit in Study 1 may imply that individuals higher on this dimension may be more likely to report $\mathrm{P}-\mathrm{J}$ fit, regardless of the job being evaluated. Individuals high on Extraversion may assume that they could get along with others in a variety of environments, and therefore report higher levels of fit. It is important, however, to recall the context of the present research: customer service jobs. Individuals higher in Extraversion may be more likely to perceive that they fit with customer service jobs in general, since these jobs involve interacting with others. Future research could be useful in determining whether these findings for Extraversion generalize beyond customer service jobs.

Significant relationships were also found in both studies between each of the JCB dimensions and subjective P-J fit. The positive relationship between beliefs regarding Customer Interaction and Organizational Interaction and $\mathrm{P}-\mathrm{J}$ fit suggests that people tend to think they would fit more with a job that affords possibilities for contact with others. Another possible explanation is that the face-to-face service job, which was given higher ratings on Customer Interaction and Organizational Interaction beliefs, is more familiar to people, and they would therefore express better fit with a job that they know more about rather than an unfamiliar job. Participants' reports of experience with each type of job provide some support for this explanation, since on average they had about 3 years of experience with face-to-face service work but less than a year of experience with telephone or e-mail service work.

The positive relationship between Complexity beliefs and P-J fit supports the idea that people prefer a job that they believe to be challenging and involving variety, rather than boring or routine. This is consistent with research on the Job Characteristics Model, which posits an association between job characteristics related to complexity and positive psychological outcomes such as satisfaction and motivation (Hackman \& Oldham, 1980). The current research suggests that this 
positive role of complexity may extend to the psychological variable of subjective $\mathrm{P}-\mathrm{J}$ fit.

Although these results suggest some main effects on perceptions of $\mathrm{P}-\mathrm{J}$ fit, especially with respect to JCBs, it is important to keep in mind that the purpose of the present research was to examine interactive effects of JCBs and personality. As noted earlier, a number of theories have noted the importance of considering both person and environment in determining behavior and have emphasized the positive outcomes of the fit between person and environment characteristics. The significant interactions discussed above underscore the usefulness of focusing on interactive effects on fit in addition to main effects.

\section{Implications}

Knowledge of the antecedents to $\mathrm{P}-\mathrm{J}$ fit should provide certain advantages to organizations attempting to recruit and retain the best possible employees. The study of $\mathrm{P}-\mathrm{J}$ fit is particularly useful in light of changes in the nature of work, since some (e.g., Leana, 2002) have observed that individuals' commitment to organizations has diminished over recent years. The fit between individuals and specific jobs (regardless of the organization in which the job exists) could play a particularly important role under these circumstances.

Moreover, past research has indicated that $\mathrm{P}-\mathrm{J}$ fit is related to a number of positive post-hire outcomes, such as satisfaction and commitment (Edwards, 1991; Saks \& Ashforth, 1997, 2002). An understanding of the process by which P-J fit perceptions are created could therefore allow organizations to attract applicants who are likely to perceive higher levels of fit and, in turn, to be satisfied and committed to their jobs. Additional research is needed to address the degree to which pre-hire fit perceptions are related to post-hire outcomes. Preliminary evidence for this relationship has been provided by Saks and Ashforth (1997, 2002), although they cautioned that fit perceptions may change once on the job. Thus, research regarding the stability of fit perceptions over time is recommended.

A related implication of the present research involves the accuracy of JCBs. Results indicated that JCBs were related to perceptions of P-J fit and interacted with personality in predicting $\mathrm{P}-\mathrm{J}$ fit. This suggests that organizations may be able to positively influence applicants' fit by influencing beliefs about job characteristics. It is important, however, to consider the accuracy of these JCBs. If an applicant accepts a job that does not turn out to have the anticipated characteristics, then evaluations of fit may be adjusted downward and the benefits of fit may not necessarily be reaped. Just as Cable et al. (2000) investigated the accuracy of individuals' pre-hire beliefs with respect to organizational 
culture, similar research is needed with regard to JCBs. Research on realistic job previews (RJPs) is also relevant to this issue. Although research has tended to show that providing realistic information prior to hire is negatively related to turnover (e.g., McEvoy \& Cascio, 1985), studies have tended not to focus on the role of realistic information in applicant attraction (see Bretz \& Judge, 1998 for an exception).

Another implication of the current research pertains to its context, customer service jobs. The importance of this context is supported by the prevalence of service jobs in the U.S. economy and the expectation of increasing employment in this area in the coming years (Hecker, 2004). Moreover, the inclusion of lower-skilled jobs in the present studies represents an extension of past research on fit, which has tended to focus on higher-skilled jobs for applicants with a college or graduate degree. Research on lower-skilled jobs is particularly important in light of the fact that as of the year 2000, approximately $75 \%$ of Americans did not hold a college degree (National Center for Education Statistics, 2003). As discussed below, future research should test the generalizability of the relationships found here to different types of jobs. Nevertheless, it is useful to examine fit in the context of jobs that suit individuals with lower levels of education, such as the jobs included in the present research.

In the literature on customer service, past research has tended to focus on face-to-face customer contact, despite the increasing use of alternative modes of communication (Bitner, Brown, \& Meuter, 2000). Findings from the current research suggest that individuals ascribe different job characteristics to jobs involving different modes of customer contact. In turn, these JCBs are related to perceptions of fit. Thus, understanding the nature of individuals' JCBs for different service jobs could allow organizations to (1) correct these beliefs, if inaccurate, or (2) appeal to applicants who are more likely to fit particular types of service jobs, based on the significant interactions found here. In addition, individuals' attitudes toward technology (i.e., their technological readiness; Parasuraman, 2000) may play a role in $\mathrm{P}-\mathrm{J}$ fit perceptions.

\section{Limitations}

As with any study, the findings from the current research should be interpreted in light of certain limitations. First, the use of a college student sample could hinder the generalizability of results to the general adult population. Note, however, that the two studies were framed in terms of part-time customer service jobs, which are held by many college students, including over $90 \%$ of the participants in the two studies reported here. In addition, the prevalence of individuals without a college education (National Center for Education Statistics, 2003) supports the 
usefulness of studying lower-skilled jobs. Nevertheless, the findings from the current research should be interpreted with caution given the reliance on a student sample and the use of part-time job scenarios. The assumption is that similar results would be found in broader adult samples as well as field samples, although with the stipulation that the nature of a job should be considered as a basis for the job characteristic belief dimensions included and the hypotheses developed.

A related concern involving generalizability pertains to the nature of the jobs included in the current research. Significant interactions were found despite the use of a narrow set of jobs, but the extent to which these findings generalize to other types of jobs merits attention. Although Study 2 manipulated the organizational context for the jobs and found no differences among conditions, all scenarios involved customer service. It is possible that some findings, such as the relationship between Extraversion and P-J fit, were driven by the customer service nature of the jobs, but this cannot be ascertained without further study of jobs in other domains.

A third potential limitation is the issue of same-source response bias, since all data were collected from the same participants. However, the hypothesis that individuals compare their evaluations of their personality to their beliefs about jobs in order to arrive at their judgments of P-J fit necessitates the study of individuals' own assessments of their personalities and their JCBs. The administration of the personality measure at a separate time from the remaining materials was an attempt to address this bias. Although this methodological approach was only employed for a portion of the Study 1 sample, it is encouraging that no differences in results were found between these participants and the remainder of the Study 1 sample.

Finally, the present research included only two antecedents to subjective P-J fit: personality and JCBs. Moreover, personality was considered in terms of the FFM, and JCBs were conceptualized along three dimensions. The selection of the JCB dimensions was based on continuity with the job characteristics literature as well as relevance to the context of the present research. However, some of the scales created to measure these dimensions in the present study demonstrated alpha reliability values that were less than ideal, and the confirmatory factor analyses on the scales in Study 2 suggest that further improvements could be made to the measures of the JCB dimensions. Indeed, measurement error may have limited the likelihood of finding support for the hypothesized interactions in this research. In addition, it is certainly possible that other dimensions of personality or JCBs would display interactive effects on subjective P-J fit, or that other variables (e.g., technological readiness) could contribute to perceptions of $\mathrm{P}-\mathrm{J}$ fit, particularly in the context of other 
types of jobs. Future research could also test hypotheses similar to those of the current research with respect to other types of fit (e.g., personality and beliefs regarding organizations predicting $\mathrm{P}-\mathrm{O}$ fit).

\section{CONCLUSION}

The finding of significant interactions between personality and JCBs in predicting subjective P-J fit provides for a better understanding of what contributes to individuals' perceptions of fit. Previous research had suggested that individual differences interact with job or organizational characteristics to predict attraction, but fit was not explicitly measured in those studies. In addition, past P-J fit research has tended to focus on outcomes rather than antecedents. The current research thus extends previous work by focusing on the interaction of personality with job characteristics beliefs in predicting subjective $\mathrm{P}-\mathrm{J}$ fit.

Organizations that seek to attract and retain the best possible employees should benefit from an understanding of what leads to individuals' perceptions of $\mathrm{P}-\mathrm{J}$ fit, since fit has been shown to lead to attraction as well as to post-hire outcomes such as job satisfaction. The current research suggests that personality and JCBs are antecedents to subjective fit. Future efforts should investigate how these variables and others contribute to fit, as well as the source and accuracy of JCBs.

\section{NOTE}

1 To test whether participants with customer service work experience had significantly different perspectives from participants with no such experience, a series of $t$-tests was performed with respect to each of the job characteristic belief dimensions and the fit scale. In these analyses of the Study 1 data, responses from participants who reported any amount of customer service work experience were compared with responses from participants who reported no customer service work experience. Results revealed no significant mean differences in responses from these two sets of participants, grouped according to work experience.

For Study 2, the same analysis was conducted. As in Study 1, there were no significant mean differences in responses from the two sets of participants who were grouped based on work experience.

\section{APPENDIX: LIST OF JOB CHARACTERISTIC BELIEF ITEMS}

In both studies, participants were asked: To what extent do you believe this job would have the following characteristics? The five-point response scale ranged from $1=$ to a little extent to $5=$ to a great extent. 


\section{Study 1}

Customer Interaction items:

You would have frequent interactions with customers.

Your work would be interactive.

Your work would be people-oriented.

Organization interaction items:

You would have frequent interactions with other people in the organization.

You would have satisfying interactions with other people in the organization.

Complexity items:

You would have challenging work.

You would have a variety of things to do at work.

You would have interesting work.

You would have complex work.

You would make use of your individual abilities.

Your work would be difficult.

You would have fast-paced work.

\section{Study 2}

Customer interaction items:

You would have frequent interactions with customers.

Your work would be interactive.

Your work would be people-oriented.

You would have satisfying interactions with customers.

Your work would be social.

Your work would be impersonal. (reverse-scored)

Organization interaction items:

You would have frequent interactions with other people in the organization.

You would have satisfying interactions with other people in the organization.

You would have a friendly work environment.

You would have good co-workers.

Complexity items:

You would have challenging work.

You would have complex work. 


\author{
Your work would be difficult. \\ Your work would require you to be knowledgeable. \\ Your work would be detailed. \\ Your work would be easy. (reverse-scored)
}

\title{
REFERENCES
}

Barrick, M. R., \& Mount, M. K. (1991). The Big Five personality dimensions and job performance: A meta-analysis. Personnel Psychology, 44, 1-26.

Blake, R. J., \& Sackett, S. A. (1999). Holland's typology and the five-factor model: A rational-empirical analysis. Journal of Career Assessment, 7, 249-279.

Bitner, M. J., Brown, S. W., \& Meuter, M. L. (2000). Technology infusion in service encounters. Academy of Marketing Science Journal, 28, 138-149.

Bowen, D. E., \& Schneider, B. (1988). Services marketing and management: Implications for organizational behavior. Research in Organizational Behavior, 10, 43-80.

Bretz, R. D., \& Judge, T. A. (1994). The role of human resource systems in job applicant decision processes. Journal of Management, 20, 531-551.

Bretz, R. D., \& Judge, T. A. (1998). Realistic job previews: A test of the adverse self-selection hypothesis. Journal of Applied Psychology, 83, 330-337.

Bryk, A. S., \& Raudenbush, S. W. (1992). Hierarchical linear models: Applications and data analysis methods. Newbury Park, CA: Sage.

Cable, D. M., Aiman-Smith, L., Mulvey, P. W., \& Edwards, J. R. (2000). The sources and accuracy of job applicants' beliefs about organizational culture. Academy of Management Journal, 43, 1076-1085.

Cable, D. M., \& DeRue, D. S. (2002). The convergent and discriminant validity of subjective fit perceptions. Journal of Applied Psychology, 87, 875-884.

Cable, D. M., \& Graham, M. E. (2000). The determinants of job seekers' reputation perceptions. Journal of Organizational Behavior, 21, 929-947.

Cable, D. M., \& Judge, T. A. (1994). Pay preferences and job search decisions: A personorganization fit perspective. Personnel Psychology, 47, 317-350.

Cable, D. M., \& Judge, T. A. (1996). Person-organization fit, job choice decisions, and organizational entry. Organizational Behavior and Human Decision Processes, 67, 294-311.

Caetano, A., \& Vala, J (1991). Implicit theories of organizing: An exploratory empirical study. Revue Internationale de Psychologie Sociale, 4, 69-81.

Costa, P.T., \& McCrae, R. R. (1992). The revised NEO personality inventory (NEO-PI-R) and NEO five-factor inventory (NEO-FFI) professional manual. Odessa, FL: Psychological Assessment Resources.

Dawis, R.V., \& Lofquist, L.H. (1984). A psychological theory of work adjustment: An individual differences model and its application. Minneapolis: University of Minnesota Press.

De Fruyt, F., \& Mervielde, I. (1999). RIASEC types and big five traits as predictors of employment status and nature of employment. Personnel Psychology, 52, 701-727.

Department of Labor (2005). America's Career InfoNet. Retrieved February 9, 2005 from http://www.acinet.org/acinet/.

Dineen, B. R., Ash, S. R., \& Noe, R. A. (2002). A web of applicant attraction: Personorganization fit in the context of web-based recruitment. Journal of Applied Psychology, $87,723-734$.

Edwards, J. R. (1991). Person-job fit: A conceptual integration, literature review, and methodological critique. International Review of Industrial and Organizational Psychology, 6, 283-357.

Frei, R. L., \& McDaniel, M. A. (1998). Validity of customer service measures in personnel selection: A review of criterion and construct evidence. Human Performance, 11, 1-27. 
Fried, Y., \& Ferris, G. R. (1987). The validity of the job characteristics model: A review and meta-analysis. Personnel Psychology, 40, 287-322.

Goldberg, L. R. (1990). An alternative "description of personality": The big-five factor structure. Journal of Personality and Social Psychology, 59, 1216-1229.

Hackman, J. R., \& Oldham, G. R. (1980). Work redesign. Reading, MA: Addison-Wesley.

Hecker, D. (2004). Occupational employment projections to 2012. Monthly Labor Review Online, 127, 80-105.

Hofstee, W. K. B., de Raad, B., \& Goldberg, L. R. (1992). Integration of the big five and circumplex approaches to trait structure. Journal of Personality and Social Psychology, 63, 146-163.

Hogan, J., Hogan, R., \& Busch, C. M. (1984). How to measure service orientation. Journal of Applied Psychology, 69, 167-173.

Holland, J. L. (1973). Making vocational choices: A theory of careers. Englewood Cliffs, NJ: Prentice-Hall.

Holland, J. L. (1996). Exploring careers with a typology: What we have learned and some new directions. American Psychologist, 51, 397-406.

Hox, J. J., \& Kreft, I. G. (1994). Multilevel analysis method. Sociological Methods and Research, 22, 283-299.

Hurley, R. F. (1998). Customer service behavior in retail settings: A study of the effect of service provider personality. Journal of the Academy of Marketing Science, 26, 115127.

Judge, T. A., \& Bretz, R .D. (1992). Effects of work values on job choice decisions. Journal of Applied Psychology, 77, 261-271.

Judge, T. A., \& Cable, D. M. (1997). Applicant personality, organizational culture, and organization attraction. Personnel Psychology, 50, 359-393.

Judge, T. A., Heller, D., \& Mount, M. K. (2002). Five-factor model of personality and job satisfaction: A meta-analysis. Journal of Applied Psychology, 87, 530-541.

Kellogg, D. L., \& Chase, R. B. (1995). Constructing an empirically derived measure for customer contact. Management Science, 41, 1734-1749.

Kraut, A. I., \& Korman, A. K. (1999). The "DELTA forces" causing change in human resource management. In A. I. Kraut \& A. K. Korman (Eds.), Evolving practices in human resource management (pp. 3-22). San Francisco: Jossey-Bass.

Kristof, A. L. (1996). Person-organization fit: An integrative review of its conceptualizations, measurement, and implications. Personnel Psychology, 49, 1-49.

Kristof-Brown, A. L., Jansen, K. J., \& Colbert, A. E. (2002). A policy-capturing study of the simultaneous effects of fit with jobs, groups, and organizations. Journal of Applied Psychology, 87, 985-993.

Lauver, K. J., \& Kristof-Brown, A. (2001). Distinguishing between employees' perceptions of person-job and person-organization fit. Journal of Vocational Behavior, 59, 454-470.

Leana, C. R. (2002). The changing organizational context of careers. In D. C. Feldman (Eds.), Work Careers: A Developmental Perspective, San Francisco: Jossey Bass.

Lievens, F., Decaesteker, C., Coetsier, P., \& Geirnaert, J. (2001). Organizational attractiveness for prospective applicants: A person-organisation fit perspective. Applied Psychology: An International Review, 50, 30-51.

McCrae, R. R., \& Costa, P. T. Jr. (1987). Validation of the five-factor model of personality across instruments and observers. Journal of Personality and Social Psychology, 52, 85-90.

McEvoy, G. M., \& Cascio, W. F. (1985). Strategies for reducing employee turnover: A metaanalysis. Journal of Applied Psychology, 70, 342-353.

Mount, M. K., \& Barrick, M. R. (1995). The big five personality dimensions: Implications for research and practice in human resources management. Research in Personnel and Human Resources Management, 13, 153-200.

Mount, M. K., Barrick, M. R., \& Stewart, G. L. (1998). Five-Factor Model of personality and performance in jobs involving interpersonal interactions. Human Performance, 11, $145-165$

Murray, H. (1938). Explorations in personality. New York: Oxford University Press. 
National Center for Education Statistics (2003). Educational attainment of persons 25 years old and over, by race/ethnicity and state: April 1990 and April 2000. Retrieved August 24, 2005 from http://nces.ed.gov/programs/digest/d03/tables/dt012.asp.

O*NET (2005). Summary Report for: 43-4051.00-Customer Service Representatives. Retrieved February 9, 2005 from http://online.onetcenter.org/.

Parasuraman, A. (2000). Technology readiness index (TRI): A multiple-item scale to measure readiness to embrace new technologies. Journal of Service Research, 2, 307320 .

Rynes, S. L. (1991). Recruitment, job choice, and post-hire consequences. In M. D. Dunnette (Eds.), Handbook of industrial and organizational psychology (pp. 399-444). 2nd edition, Palo Alto, CA: Consulting Psychologists Press.

Saks, A. M., \& Ashforth, B. E. (1997). A longitudinal investigation of the relationships between job information sources, applicant perceptions of fit, and work outcomes. Personnel Psychology, 50, 395-426.

Saks, A. M., \& Ashforth, B. E. (2002). Is job search related to employment quality? It all depends on fit. Journal of Applied Psychology, 87, 646-654.

Schneider, B. (1987). The people make the place. Personnel Psychology, 40, 437-454.

Schneider, B., Goldstein, H. W., \& Smith, D. B. (1995). The ASA framework: An update. Personnel Psychology, 48, 747-773.

Schneider, B., Smith, D. B., \& Goldstein, H. W. (2000). Attraction-selection-attrition: Toward a person-environment psychology of organizations. In W. B. Walsh, K. H. Craik \& R. H. Price (Eds.), Person-environment psychology: New directions and perspectives (pp. 61-85). Mahwah, NJ: Lawrence Erlbaum Associates.

Soteriou, A. C., \& Chase, R. B. (1998). Linking the customer contact model to service quality. Journal of Operations Management, 16, 495-508.

Spence, M. (1973). Job market signaling. Quarterly Journal of Economics, 87, 355-374.

Sproull, L. (1981). Beliefs in organizations. In P. Nystrom \& W. Starbuck (Eds.), Handbook of organizational design: Vol. 2 (pp. 203-244). London: Oxford University Press.

Stone, E. F., \& Gueutal, H. G. (1985). An empirical derivation of the dimensions along which characteristics of jobs are perceived. Academy of Management Journal, 28, 376-396.

Terborg, J. R. (1981). Interactional psychology and research on human behavior in organizations. Academy of Management Review, 6, 569-576.

Tokar, D. M., \& Swanson, J. L. (1995). Evaluation of the correspondence between Holland's vocational personality typology and the five-factor model of personality. Journal of Vocational Behavior, 46, 89-108.

Turban, D. B., \& Keon, T. L. (1993). Organizational attractiveness: An interactionist perspective. Journal of Applied Psychology, 78, 184-193. 